\title{
The Pulfrich spatial frequency phenomenon: a psychophysical method competitive to visual evoked potentials in the diagnosis of multiple sclerosis
}

\author{
E U G E N E R. W I S T, M. HE N N R I C I, A N D J. D I C H G A S \\ From the Department of Neurology, University of Freiburg, Freiburg, West Germany
}

SUMMARY The results of a study in which visual evoked responses (VERs) and a modified Pulfrich method were compared showed that both methods are very effective for the diagnosis of multiple sclerosis. With VERs, $97 \%$ of the multiple sclerosis cases were diagnosed correctly, while the corresponding value for the Pulfrich method was $93 \%$. In contrast to VERs, the Pulfrich method allows only measurement of latency differences between the two visual pathways. This method involves measuring the speed required to cause a shift in the apparent depth location of a large, moving, striped pattern observed with a neutral density filter over one eye. A pathological transmission time was inferred when the patients observed a shift in the depth of the moving pattern either without any filter at all or with a filter whose attentuation was no more than $0.2 \mathrm{log}$ units. A further criterion for pathology was a difference of more than $10 \%$ between the two eyes in the retinal speed required for a depth displacement using a $1.5 \mathrm{log}$ unit filter. This test requires about 15 minutes, and can be carried out by a technical assistant.

The use of visually evoked responses (VERs) has received increasing attention for the clinical diagnosis of multiple sclerosis (McDonald and Halliday, 1977). Since the studies of Halliday et al. (1972) and Asselman et al. (1975), the latencies of the average cortical responses evoked by checkerboard pattern reversal stimulation have been known to be abnormally long in the affected eye of patients with optic neuritis. Although optic nerve lesions could be detected with this method in some patients suspected of multiple sclerosis even in the absence of a history of visual impairment or ophthalmological abnormalities, the incidence of a delay of the major positive potential was significantly smaller for patients showing a more slight and chronic course of the disease than for cases classified as definite using the diagnostic criteria of McAlpine et al. (1972). As recently shown by Hennerici et al. (1977), the sensitivity of the VER method could be improved by use of a small square stimulus placed in the visual axis (foveal stimulation). This was suggested to be because of a more common demyelination of fibres

\footnotetext{
Address for reprint requests: Professor Eugene R. Wist, Psychologisches Institut der Universität Düsseldorf, Universitätsstrasse 1 4000 Düsseldorf, West Germany.

Accepted 9 May 1978
}

originating from the macular region than of fibres outside the central $3-4^{\circ}$. Nevertheless, both stimuli were considered necessary for the optimal detection of demyelination since, in a very few cases, only the checkerboard stimulus was effective.

In addition to VERs, at least two psychophysical means for the diagnosis of multiple sclerosis have been used. Whereas the VER method measures increased transmission times in each visual pathway independently, the psychophysical procedures are designed to measure interocular latency differences. Heron et al. (1974) and Regan et al. (1976) successfully used a relatively complicated psychophysical procedure for the measurement of perceptual latencies in optic neuritis. Frisen et al. (1973) suggested the Pulfrich stereophenomenon (Pulfrich, 1922) as a means of diagnosing multiple sclerosis, and Rushton (1975) presented data from a clinical study involving 35 patients with multiple sclerosis in which a variation of the Pulfrich effect was employed. However, in his study, $60 \%$ of the multiple sclerosis patients failed to show an abnormal Pulfrich effect. Furthermore, $12 \%$ of the 23 patients without multiple sclerosis showed an abnormal Pulfrich effect.

On the basis of our finding that the Pulfrich effect is exaggerated when a moving periodic, ver- 
tical stripe pattern instead of a point of light, such as was used in the Rushton study, is viewed (Wist et al., 1977), we predicted and found a considerable improvement in the sensitivity of the Pulfrich effect as an indicator of abnormal transmission times in the visual pathway. In the earlier study we found that the amount of displacement in depth of a moving periodic pattern of alternate light and dark stripes was a function not only of the density of the filter placed before one eye and the angular speed of pattern movement, but also of the spatial frequency-the greater the spatial frequency for a constant filter density and angular speed, the greater the depth displacement (Wist et al., 1977). Furthermore, the depth displacement for a periodic pattern was greater than that obtained for a single stripe. This latter condition corresponds most closely to the usual way of demonstrating the Pulfrich effect.

For the purpose of the present study we reasoned, therefore, that the use of a large periodic pattern of appropriate spatial frequency would magnify depth displacement. Consequently, our procedure might be a more sensitive one for the measurement of increased transmission times in multiple sclerosis because even small transmission time increases in one visual pathway, acting in a manner equivalent to a low density filter over one eye, would lead to larger apparent depth displacement via the spatial frequency effect, than would be the case with a pendulum or spot of light. In addition, the use of a large stimulus area facilitates the detection of transmission delays resulting from demyelination or oedema over a larger crosssection of the visual pathway. The advantages of this method are that it is simple, rapid, does not require expensive equipment, and, with the improvements made, its sensitivity is comparable to that of VERs.

\section{Patients and methods}

Twenty-seven multiple sclerosis patients ranging between 21 and 70 years of age were studied. According to the diagnostic criteria of McAlpine et al. (1972) these patients were classified into three categories: definite diagnosis $(n=13)$, probable diagnosis $(n=5)$, and possible diagnosis $(n=9)$. An additional neuro-ophthalmological examination was performed to exclude other possible diseases causing an increase in transmission time in the visual pathway. In addition, 32 control subjects were studied, 16 of whom were patients with diseases other than multiple sclerosis, and 16 of whom were normal subjects. The age range of this control group was from 14 to 65 years. Two sub- jects were excluded because they lacked stereoscopic vision as measured by the Pulfrich test itself. Because of the low spatial frequency of the moving stripe pattern, visual acuity was not critical.

\section{PULFRICH EFFECT}

The patient sat in an upholstered chair fitted with a head support with his eyes at a distance of 79.5 $\mathrm{cm}$ from a cylindrically shaped screen which subtended a visual angle of $165^{\circ}$ in width and $100^{\circ}$ in height (Fig. 1). A Tonnies Optokinetic Stimulator was used to project a regular pattern of horizontally moving vertical, alternate dark and light stripes onto the screen. The angular width of the dark stripes was $6^{\circ}$ while that of the light ones was $12^{\circ}$. Thus the spatial period was $18^{\circ}$ and the corresponding spatial frequency was 0.055 cycles/degree. The luminance of the light stripes was $7.95 \mathrm{~cd} / \mathrm{m}^{2}$ while that of the dark ones was $1.75 \mathrm{~cd} / \mathrm{m}^{2}$. Consequently, the contrast ratio was 0.64 . A small dark disc $0.5^{\circ}$ in diameter affixed to the screen in the patient's median plane at eye level facilitated depth discrimination, and a larger $\widetilde{D}$ dark disc $1^{\circ}$ in diameter suspended by fine threads $\stackrel{\oplus}{\sim}$ $15 \mathrm{~cm}$ in front of the screen $3^{\circ}$ to the left of the reference point served as a fixation point.

The procedure was designed to determine for each of two filter densities the angular speed of pattern movement required to produce a forward shift in the perceived depth of the moving pattern up to the marker disc as shown in Fig. 2. The filters provided either 0.2 or $1.5 \mathrm{log}$ units of attenuation. The lower attenuation filter was not sufficiently dense to produce a Pulfrich depth displacement in normal subjects but did produce depth displacement in some multiple sclerosis patients. The density of the $1.5 \mathrm{log}$ unit was sufficient to yield a clear Pulfrich depth displacement for both patients and normals.

First, the patient was asked to fixate upon the marker disc. Next he was asked to observe the stripes as they moved across the screen first in one direction and then in the other. The patient was told that the stripes could appear to be moving either upon the screen, or in front of or behind its surface. Two trials were given without any filter at all during which the stripe speed was gradually increased by the experimenter from 0 to 60 degrees per second. The patient was required to state whether the stripes appeared behind, upon, or in front of the screen. Next, two trials were given with the 0.2 filter, once held over the left eye with the stripes moving to the left, and once held over the right eye with stripes moving to the right. The last two practice trials were conducted in the same manner as the previous two, except that the $1.5 \mathrm{log}$ 


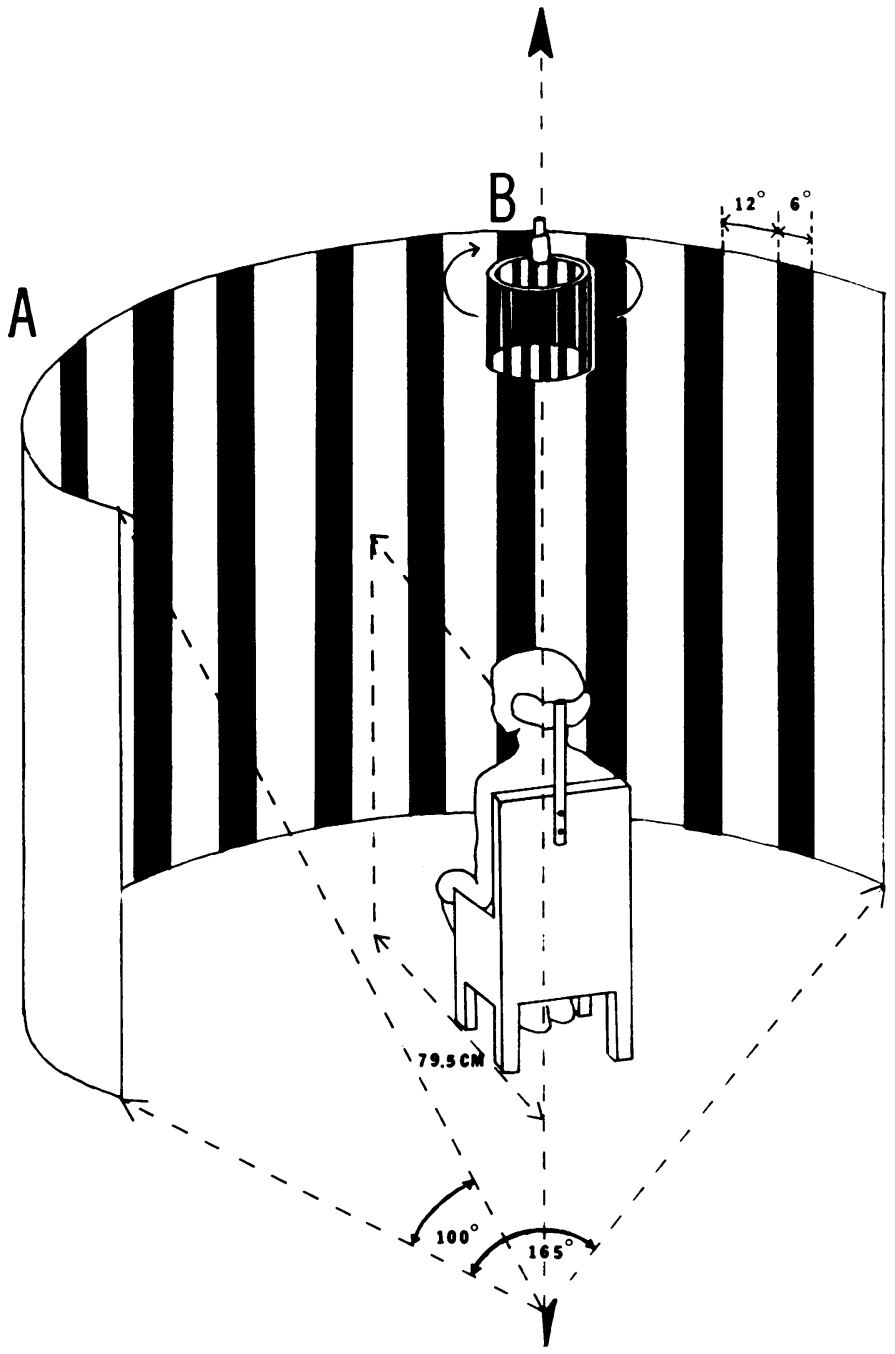

Fig. 1 Stimulus display with relevant dimensions. The dot in the subject's median plane represents the reference point. Also shown are the projection screen $(A)$ and the optokinetic stimulator (B).

unit filter was used instead. These latter conditions produce apparent forward displacement of the moving stripes in normal subjects, but subjects were not informed that this is the case. Conditions resulting in apparent rearward displacement were given at the end of the test as a control. But for both patients and normal subjects this effect was often difficult to observe since it involves seeing the stripes moving behind an opaque screen.

After this practice series, 10 trials were conducted with the 0.2 filter held alternatively over the left and right eyes, if the patient had seen a Pulfrich depth displacement with this filter during the practice series. For each trial, the angular speed was slowly increased by the experimenter until the perceived depth plane of the moving stripes shifted forward to the distance of the marker disc as shown in Fig. 2. This speed was then recorded. Another 10 trials were subsequently conducted in the same way, this time using the $1.5 \log$ unit filter.

All Pulfrich tests were carried out blindthat is, without previous knowledge of the diagnosis of any of the patients. Only after the completion of the study were the diagnoses based on the Pulfrich test compared with the actual diagnoses and the VER results. Moreover, in order to evaluate the applicability of this method for routine clinical use, over half of the subjects were tested by a laboratory technician. She did not 


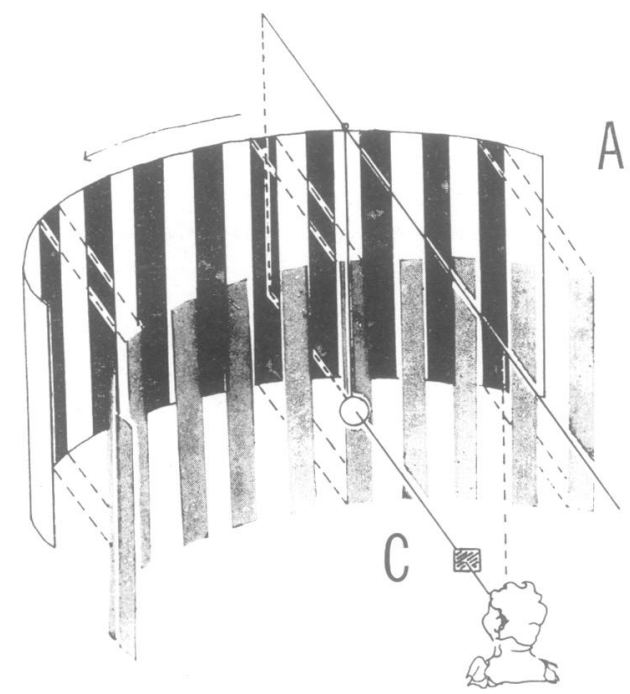

Fig. 2 Illustration of the apparent forward displacement to the criterion distance in the plane of the fixation disc $(B)$ when a filter $(C)$ is placed over the left eye, and movement of the projected stripes is to the left on the screen $(A)$.

know the diagnosis of the patients and was also unaware of the possible diagnostic significance of the Pulfrich method.

\section{VER RECORDING}

The average visual evoked responses were recorded for two different stimulus conditions. A television monitor (Philips) producing 625 lines and 50 fields per second was located $150 \mathrm{~cm}$ in front of the patient who was instructed to fixate a dark spot at the centre of the monitor throughout each run. One hundred and twenty-eight successive responses were averaged from each eye for each of two series of stimuli.

In the first series a black and white checkerboard pattern which subtended $20^{\circ}$ of the visual field was produced on the monitor. At intervals of $800 \mathrm{~ms}$ the black squares $\left(0.3426 \mathrm{~cd} / \mathrm{m}^{2}\right)$ became white $\left(51.39 \mathrm{~cd} / \mathrm{m}^{2}\right)$ and the white, black. This reversal of contrast was accomplished by means of a time pulse generator. The checks subtended a visual angle of $1^{\circ} 10 \mathrm{~min}$. The pulse triggering each contrast reversal was also used to trigger a Nicolet computer in order to average the EEG recorded from the scalp electrode which was located $6 \mathrm{~cm}$ above the inion in the midline and referred to a common linked ear reference.

In the second series a bright $\left(130 \mathrm{~cd} / \mathrm{m}^{2}\right)$, small square was displayed foveally on a diffuse monitor background illumination $\left(3.1 \mathrm{~cd} / \mathrm{m}^{2}\right)$. This stimulus was located on the visual axis in the centre of the monitor, and subtended a visual angle of $45 \mathrm{~min}$ in the central retina (foveal stimulation). It was presented repeatedly with an on-time of 500 and an off-time of $500 \mathrm{~ms}$. Each point of the curve could be analysed digitally in terms of amplitude and latency with a time resolution of one millisecond. Latencies refer to the time from stimulus onset to the peak of the major positive potential. For both methods the same significance criteria as to "normal" or "pathological" were assessed: (1) lengthening of the latency beyond the normal upper limit (mean +3 SD); (2) excessive difference in latency between the two eyes even if the absolute latencies in both eyes were normal; (3) amplitude and shape differences in the VERs deviating from normal. These were only taken into account if abnormalities with the first two criteria were found. Further details on the VER method are given by Hennerici et al. (1977).

\section{Results}

The mean angular speed in degrees/second required to shift perceptually the motion plane of the moving stripes to the distance of the marker disc (as shown in Fig. 2) was calculated for each eye and for each filter and used as a measure of the temporal delay in the visual pathway. If the filters introduced equal delays, then the angular speeds required for the shift in depth should be equal. The simplest way to express this relation was the formation of the speed ratio:

$\frac{\bar{\omega}_{\mathrm{LE}}}{\bar{\omega}_{\mathrm{RE}}}$ where $\bar{\omega}=$ the mean angular speed in degrees/ second when the filter covered the left eye (LE) or the right eye (RE).

When this ratio is equal to 1.00 , the angular speeds required for the two eyes are equal, and, therefore, the perceptual delays are equal in the two visual pathways. When the ratio is less than 1.00 , less speed is required for the left than for the right eye, and, therefore, a greater delay exists in the left visual pathway. When this ratio is greater than 1.00 , the speed required for the left eye is greater than for the right, and, therefore, a greater delay exists in the right visual pathway. As determined from our previous study (Wist et al., 1977), this ratio falls within the limits of $1.00 \pm 0.10$ for normal subjects (mean $=1.00$ ). The mean angular speed required to produce a forward displacement of apparent depth of the moving stripes for all subjects for the $1.5 \mathrm{log}$ unit filter was 42 degrees/ second $(\mathrm{SD}=12.8)$. Intrasubject variability in the angular speed required for apparent depth displacements was relatively small for both multiple 
sclerosis and non-multiple sclerosis patients $(\mathrm{SD})=$ 3.92 and 2.96 respectively).

PATIENTS WITH MLLTIPLE SCLIEROSIS

An abnormal Pulfrich effect was judged to exist if either of two criteria were met: (1) the patient showed a Pulfrich effect with the 0.2 filter in at least one eye. (2) the patient produced a speed ratio which was outside the normal limits of 1.00 \pm 0.10 . For the purposes of this study. the diagnosis of multiple sclerosis was made in both instances even though other neuro-ophthalmological or neurological disorders may also result in increased neural transmission times (Hennerici et al.. 1977). In fact, one patient not included in this siudy showed both increased VER latencies and abnormal Pulfrich effects. He suffered from myoclonus epilepsy which is known to cause neural transmission delays.

For the diagnosis of multiple sclerosis by means of the VERs. the main criterion was the existence of a latency of the major positive potential greater than the mean normal latency plus three standard deviations. For foveal stimulation this meant a latency of over $130 \mathrm{~ms}$, and for the checkerboard pattern a latency of over $112 \mathrm{~ms}$ in either eye. In addition, an interocular difference in latency exceeding $9 \mathrm{~ms}$ was regarded as pathological for both VERs, cven when the absolute latency for the two eyes was within normal limits.

The distribution of Pulfrich ratios for three subclasses of patients is shown in Fig. 3. In panel A the distribution of speed ratios is shown for those patients obtaining a Pulfrich effect with the 0.2 filter $(n=13)$. Seventy per cent of the multiple sclerosis patients were able to observe a Pulfrich depth displacement without any filter. For most of these patients the displacement of the stripes from the screen was only several centimetres even with the optimal speed, but for a few, the displacement was as large as shown in Fig. 2. In these cases. one could be confident of the existence of pathology in
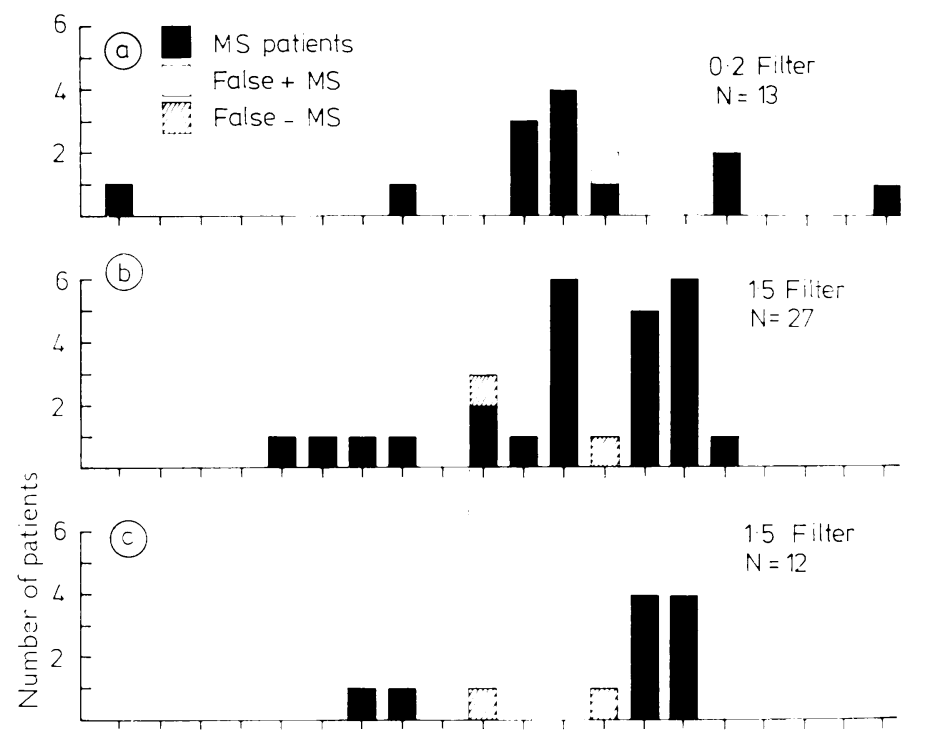

Fig. 3 Frequency distributions of the Pulfrich speed ratios $\left(\bar{\omega}_{L E} / \omega_{R E}\right)$. The numbers on the abscissa represent the lower limits of intervals of 0.05 units. The vertical dashed lines in the centre represent the normal limits of $1.00 \pm 0.10$. (a) Speed ratios obtained for 13 multiple clerosis patients with the zero filter. With one exception, a false positive indicated in white, only multiple sclerosis patients showed a zero filter effect. Not included are two additional multiple sclerosis patients who showed a 0.2 filter effect for the right eye only. (b) Ratios for all 27 multiple sclerosis patients ob:ained with the 1.5 filter. Of those falling within the normal limits all but two showed a 0.2 filter effect as well, and were, therefore, diagnosed correctly as multiple sclerosis. The two exceptions were false negatives. (c) Ratios obtained with the 1.5 filter for the 12 multiple sclerosis patients not showing a 0.2 filter effect. All except the two false negative cases also shown in (b) are outwith the normal limits. (d) Normal ratios for the 31 control subjects without multiple sclerosis obtained with the 1.5 filter all are within the normal limits except for the false positive multiple sclerosis case shown in white.

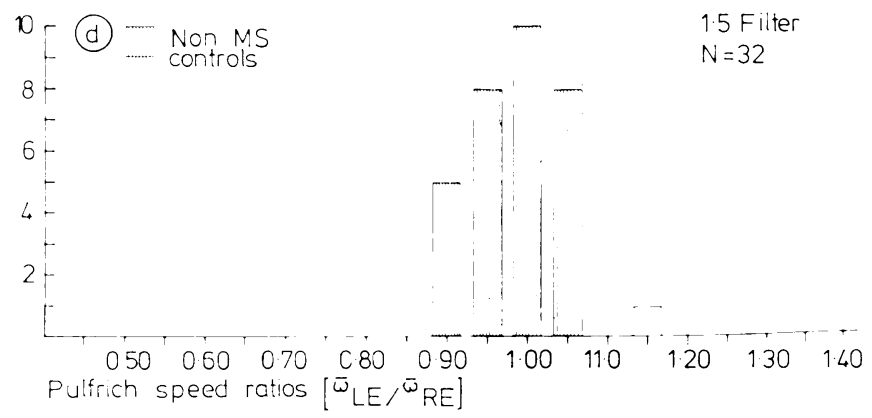


the first minute or two of the test before any quantitative measurements had been obtained with either filters.

The two patients who obtained the 0.2 filter effect with the right eye only are not shown here because it was not possible to calculate ratios for them. As indicated, only one of these patients, who suffered from a thyroid carcinoma, was falsely diagnosed as multiple sclerosis on the basis of showing a 0.2 filter effect. In panel B the frequency distribution of ratios for the 1.5 filter data is shown for all 27 multiple sclerosis patients. All but two of the patients having ratios with the 1.5 filter within the normal limits showed a 0.2 filter cffect as well, and were, therefore, correctly diagnosed as having multiple sclerosis. These two patients are indicated by cross-hatching in panel B. Both were cases of definite multiple sclerosis. One had a speed ratio of 0.90 which is at the lower limit of the normal range, while the other had a ratio of 1.06 . In panel $C$ the 1.5 filter data of only those patients $(n=12)$ who experienced no 0.2 filter effect are shown. It can be seen that all but two of these ratios lie outside the normal limits. As seen from Figs. 3, 5, and 6, both the psychophysical and electrophysiological methods show a bias toward longer latencies in the right eye for multiple sclerosis patients. This fact, although noteworthy, cannot be accounted for at present. All were correctly diagnosed as multiple sclerosis except for the two false negatives whose correct diagnosis was definite multiple sclerosis.

Figure 4 is a scatter plot of the relationship between the ratios obtained with the 0.2 filter for the patients in panel A of Fig. 3 and the ratios obtained with the 1.5 filter for these same patients. Although this figure shows a positive relation between the ratios obtained under the two conditions, the correlation coefficient was only +0.52 because of two deviant patients.

Figure 5 is a plot of the relationship between the 1.5 filter Pulfrich ratios and checkerboard VER latency differences for the 27 multiple sclerosis patients. Although there was a high degree of correspondence between the correct detection of multiple sclerosis for at least the foveal VER and Pulfrich methods ( 25 of 27 cases), the extent of correspondence was much poorer concerning lateralisation of the greater delay. For the checkerboard VER, the two methods agreed upon which visual pathway showed the greater delay or showed no significant difference between the two pathways in 10 of the 27 cases as shown by the points in the shaded areas. The Pulfrich method detected latency differences in seven cases in which the VER method detected none. Conversely, VER

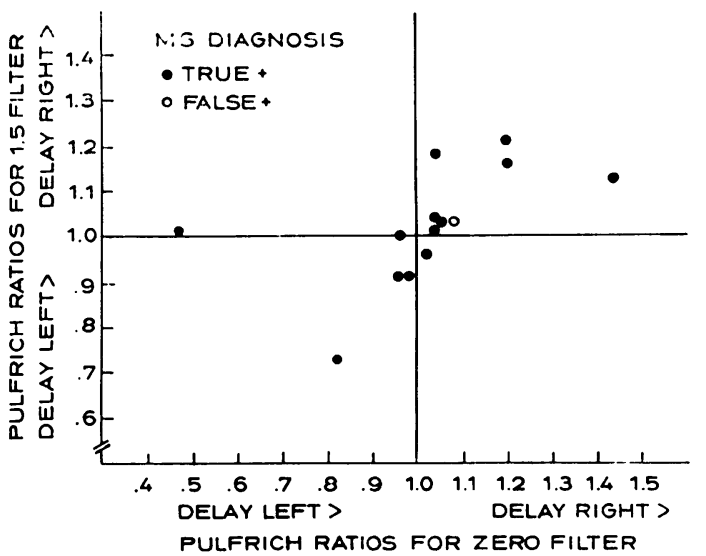

Fig. 4 Relationship between the Pulfrich speed ratios obtained for the 0.2 filter (abscissa) and the 1.5 filter (ordinate) for the 13 multiple sclerosis patients and one false positive in panel (a) of Fig. 3. The positive relationship between the two sets of results demonstrates their consistency.

latency differences were detected in six cases in which the Pulfrich ratio method detected none (but these cases did show 0.2 filter effects).

Figure 6 shows the same relationship, this time for the foveal stimulation VERs. Agreement was found in 11 cases. Furthermore, the Pulfrich ratio method detected significant lateralisation in four cases in which the VERs detected none, while the VERs detected eight cases of lateralisation not detected by the Pulfrich method. In both Figs. 5 and 6 there appears to be no systematic relationship between the degree of certainty of the diagnosis of multiple sclerosis and the extent of agreement on lateralisation between the VER and Pulfrich methods. An interesting observation was the finding in one of the multiple sclerosis patients of the recovery of both a pathological foveal VER latency and an abnormal 1.5 filter speed ratio after cortisol treatment. It should be noted also that agreement on the presence or absence of lateralisation between the two different VER stimuli was seen in only 14 of the 27 cases because of the greater sensitivity of the foveal method to lateralisation. However, in only two cases was lateralisation reversed for the two VER stimuli. For another two, lateralisation was only indicated by one of the stimuli. The remaining nine cases were pathological with only one of the two stimuli.

Figure 7 gives a summary of the results for both the VER and Pulfrich methods separately and combined in terms of the percentages of multiple sclerosis patients correctly diagnosed. It shows that the foveal VER was considerably better than the 


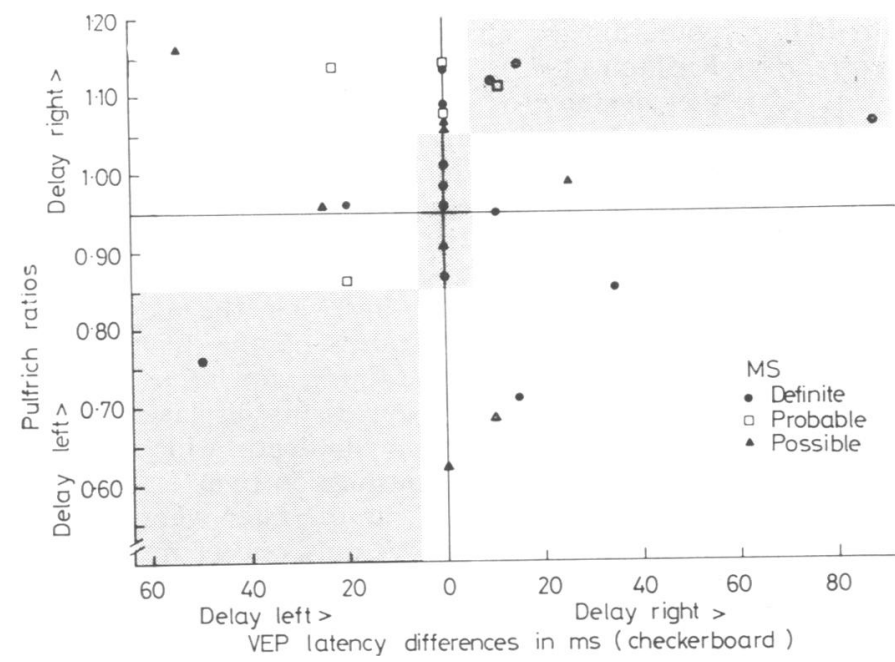

Fig. 5 Relationship between interocular checkerboard VER differences (abscissa) and Pulfrich speed ratios for the 1.5 filter ordinate for all multiple sclerosis patients. Points within the shaded areas indicate agreement, and points outside the shaded areas indicate disagreement between the two methods.

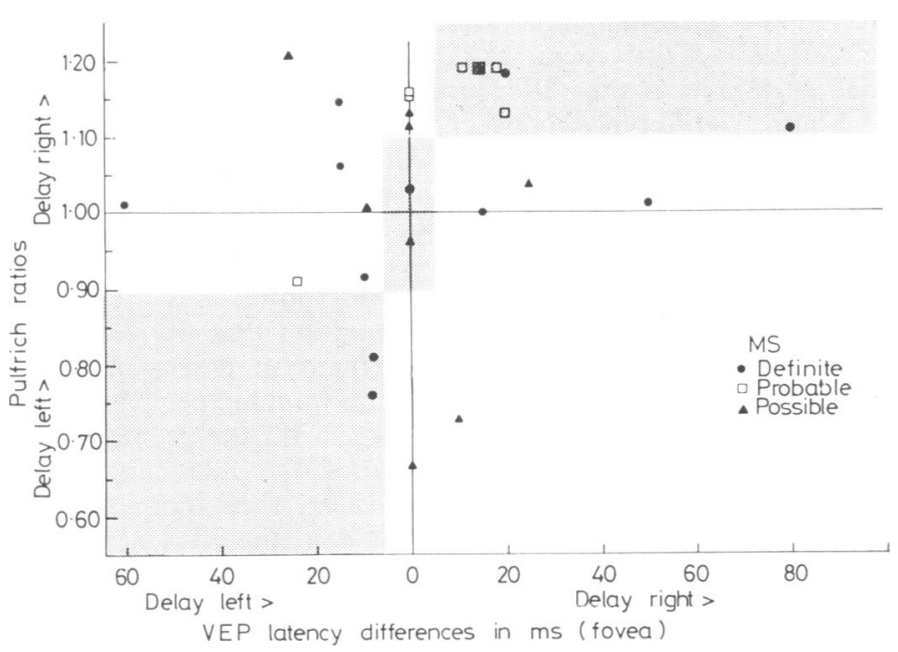

Fig. 6 Same relationship shown in Fig. 5 for foveal VERs.

checkerboard VER in that $93 \%$ of the cases of multiple sclerosis were correctly diagnosed as opposed to only $62 \%$ for the latter method. When the results for both methods were combined the percentage of correct diagnoses rose to $97 \%$. Figure 7 shows further that if only the criterion of showing a 0.2 filter effect had been used only $56 \%$ of the multiple sclerosis patients would have been diagnosed correctly. Moreover, if only the criterion of a $1.5 \mathrm{log}$ unit filter ratio outside the normal limits had been applied, only $59 \%$ of the multiple sclerosis patients would have been diagnosed correctly. When both criteria are combined, however, the percentage of correct diagnoses was $93 \%$, virtually the same as obtained with the combined foveal and checkerboard VERs. When both the VER and Pulfrich methods were combined to

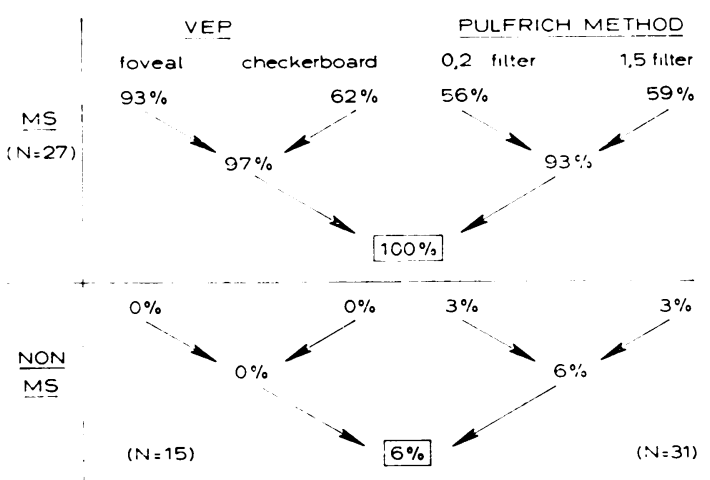

Fig. 7 Percentages of subjects showing pathological results for the VER and Pulfrich methods. Arrows indicate that by combining criteria and methods the percentage of correct detections increases. 
determine the diagnosis of multiple sclerosis $100 \%$ of the cases of that disease were correctly diagnosed.

PATIENTS WITHOUT MULTIPLE SCLEROSIS

Panel D of Fig. 3 shows the distribution of Pulfrich ratios obtained with the 1.5 filter for the 32 subjects without multiple sclerosis. They all fall within the normal range of $1.00 \pm 0.10$ except for one false positive case (narcolepsy). Included in this group of subjects without multiple sclerosis werc 16 patients with spinal cord or lumbar diseases and others with headache or minor cerebral abnormalities other than multiple sclerosis. As shown at the bottom right of Fig. 7, two of the 32 subjects without multiple sclerosis $(6 \%)$ produced Pulfrich ratios indicating pathology. One was the patient with narcolepsy mentioned above whose abnormal 1.5 filter ratio is illustrated in panel D of Fig. 3 . The other was the patient with a thyroid carcinoma who showed an abnormal 0.2 filter effect only, and whose ratio is included in panel A of Fig. 3. As shown at the bottom left of Fig. 7, none of the 15 patients without multiple sclerosis for whom VER recordings were made showed pathological results.

\section{Discussion}

The results obtained with the Pulfrich method in this study were considerably better than those obtained by Rushton (1975). Using the joint criterion of 0.2 filter effect and/or a Pulfrich ratio outside the normal limits, $93 \%$ of the multiple sclerosis patients were diagnosed correctly as opposed to only $40 \%$ in the Rushton study. Furthermore, even if a single criterion had been employed, the percentages of correct diagnoses would have been greater: $56 \%$ on the basis of a zero filter effect alone and $59 \%$ on the basis of an abnormal ratio with the 1.5 filter only.

This greater success may be attributable to three factors. Firstly, a periodic pattern was used with a spatial frequency in the range found in an earlier study to increase the amount of apparent depth displacement produced by a given filter density and angular speed of stimulus movement (Wist et al., 1977). We found in this study approximately $50 \%$ more depth displacement than that obtained from a very low frequency pattern or from a single stripe. By use of the periodic pattern, latency differences which are too small to cause a suprathreshold effect with the traditional Pulfrich method are magnified.

Secondly, a very large, continuous, moving stimulus field was used, in contrast to the single point moving through a 6 degree field used by Rushton (1975). This had the effect of increasing the vividness of the stereoscopic depth displacement. An entire striped surface was seen, moving continuously, and advancing toward the subject in depth with increasing angular speed until it appeared equidistant to the fixation disc. This characteristic of the display made the psychophysical task of detecting when the moving surface reached the distance of the fixation disc relatively easy. Interestingly, for some multiple sclerosis patients this apparently displayed surface appeared somewhat wavy in depth, which suggests local differences in transmission time.

Thirdly, the use of the 0.2 filter whose density was sufficiently low that only one of the patients without multiple sclerosis was able to detect depth displacement when it was placed before one eye, contributes significantly to the success of this method. The most plausible explanation for its effectiveness would be that while in normal subjects this filter produced a subthreshold latency difference between the two eyes, combined with the neural transmission time increase, it yielded depth displacements which were clearly suprathreshold. All but two of the 15 multiple sclerosis patients showing a 0.2 filter effect exhibited a Pulfrich effect when this filter was held over either eye. On the assumption that the zero filter results in a small increment in perceptual delay of a constant amount, one would expect that when it was held before the eye with the greater transmission delay, addition of the filter and transmission delays would occur, thus increasing the total latency. When, however, the filter was placed before the other eye, it would also increase the total latency of that eye but would at the same time reduce the latency difference between the two visual pathways. The result should be a minimal Pulfrich effect or none at all if equalisation of the latencies in the two pathways resulted. Yet for nine of the 15 multiple sclerosis patients showing a 0.2 filter effect the Pulfrich depth displacement was equally great for the two eyes.

This paradox might be explained by the hypothesis that the effect of the 0.2 filter is not simply to add a constant increment of perceptual delay, but rather that the delay induced by this filter is proportional to the extent of transmission delay resulting from demyelination, the greater the transmission delay the greater the effective delay of the 0.2 filter. This would account for the fact that patients without multiple sclerosis do not show a 0.2 filter effect, and would, at the same time, explain why the 0.2 filter produced a Pulfrich depth displacement when held before either eye by 
multiple sclerosis patients. This hypothesis could be supported if it were found that the VER latency increase resulting from the 0.2 filter was greater for multiple sclerosis patients than for normal subjects. Such a study is currently being conducted.

In spite of the 0.2 filter's significance for a positive diagnosis of some cases of multiple sclerosis, it is not clear at this point why $41 \%$ of the multiple sclerosis patients failed to show a 0.2 filter effect. It was not possible to discriminate between patients showing 0.2 filter effects and those showing none on the basis of either absolute VER latencies or VER latency differences.

Other discrepancies exist in the comparison of VER and Pulfrich methods. As was described in Figs. 5 and 6 , the correlation between the predicted lateralisation for the two methods is very low. Why this should be the case is not understood at present. It may have to do with an adaptation effect which occurs for the multiple sclerosis patient in his interaction with moving objects or self-generated relative motion of the visual surround in his everyday environment. The extent of this adaptation would presumably depend upon the progress of the disease and its duration. As long as this adaptation were incomplete, abnormal Pulfrich effects could still be possible, especially as accentuated by the use of periodic patterns. Such an adaptation is unlikely to occur with respect to VER latencies. While VERs indicate lateralisation fairly reliably in patients with recent retrobulbar neuritis, this was not the case for five of six such patients for the Pulfrich method. Nevertheless, the method detected an abnormality, and, therefore, is of diagnostic value.

In conclusion, our data show clearly that the Pulfrich method, when modified to take advantage of the spatial frequency effect on depth displacement, is a very sensitive diagnostic indicator of abnormal transmission times in the visual pathway. Although its false positive rate was higher, it was by itself able to discriminate correctly almost the same percentage of multiple sclerosis patients as did the foveal VERs, and was far better than the usual checkerboard stimulation method. Furthermore, as indicated by the data, when used in conjunction with the foveal and checkerboard VER data, perfect discrimination between patients with and those without multiple sclerosis resulted in our sample. The fact that the psychophysical diagnosis of multiple sclerosis versus nonmultiple sclerosis was made without knowledge of the actual diagnosis lends credence to this method. Finally, the method is simple and straightforward enough so that a laboratory technician can con- duct the test in 15 minutes or less. In the present study, the data of 35 of the 59 subjects tested were collected in this way. There was no significant difference in the success rate of the patients so tested and the remainder tested by the first author. The method is also simple enough to be used with the average patient who, in our experience, has no difficulty in understanding the task. The necessary equipment is available in any routine laboratory for electronystagmography. Only the purchase of two filters is required.

This work was supported by the Deutsche Forschungsgemeinschaft, SFB 70, and began while Professor Wist was the recipient of the US Senior Scientist Award from the Alexander von Humboldt Stiftung. We thank Mrs U. Rommelt for her valuable technical assistance.

\section{References}

Asselman, P., Chadwick, D. W., and Marsden, C. D. (1975). Visual evoked responses in the diagnosis and management of patients suspected of multiple sclerosis. Brain, 98, 261-282.

Frisen, L., Hoyt, W. F., Bird, A. C., and Weale, R. A. (1973). Diagnostic uses of the Pulfrich phenomenon. Lancet, 2, 385-386.

Halliday, A. M., McDonald, W. I., and Mushin, J. (1972). Delayed visual evoked responses in optic neuritis. Lancet, 1, 982-985.

Hennerici, M., Wenzel, D., and Freund, H.-J. (1977). The comparison of small-size rectangle and checkerboard stimulation for the evaluation of delayed visual evoked responses in patients suspected of multiple sclerosis. Brain, 100, 119-136.

Heron, J. R., Regan, D., and Milner, B. A. (1974). Delay in visual perception in unilateral optic atrophy after retrobulbar neuritis. Brain, 97, 69-78.

McAlpine, D., Lumsden, C. E., and Acheson, E. D. (1972). Multiple Sclerosis: a Reappraisal: Livingstone: Edinburgh.

McDonald, W. I., and Halliday, A. M. (1977). Diagnosis and classification of multiple sclerosis. British Medical Bulletin, 33, 4-8.

Regan, D., Milner, B. A., and Heron, J. R. (1976). Delayed visual perception and delayed visual evoked potentials in the spinal form of multiple sclerosis and in retrobulbar neuritis. Brain, 99, 43-66.

Rushton, D. (1975). Use of the Pulfrich pendulum for detecting abnormal delay in the visual pathway in multiple sclerosis. Brain, 98, 283-296.

Pulfrich, C. (1922). Die Stereoskopie im Dienste der isochromen und heterochromen Photometrie. Naturwissenschaften, 10, 553-564.

Wist, E. R., Brandt, Th., Diener, H.-C., and Dichgans, J. (1977). Spatial frequency effect on the Pulfrich stereophenomenon. Vision Research, 17, 391-397. 